\title{
Toward centrality determination at NICA/MPD
}

\author{
A.S. Galoyan ${ }^{1, \star}$ and V.V. Uzhinsky ${ }^{2}$ \\ ${ }^{1}$ VBLHEP, Joint Institute for Nuclear Research, Dubna, Russia. \\ ${ }^{2}$ LIT, Joint Institute for Nuclear Research, Dubna, Russia.
}

\begin{abstract}
Geometrical properties of nucleus-nucleus interactions at various centralities are calculated for the NICA energy range. A modified version of the Glauber Monte Carlo simulation code has been used for the calculations. It is shown that the geometrical properties of nucleus-nucleus interactions at the energies $5-10 \mathrm{GeV}$ (NICA/MPD) and at energy $200 \mathrm{GeV}$ (RHIC) are quite close to each other. A possible determination of centrality at NICA/MPD experiment using calculations of various Monte Carlo event generators are considered.
\end{abstract}

\section{Introduction}

As it is known, a new accelerator facility - the Nuclotron based Ion Collider facility (NICA) at JINR (Dubna) will start to operate in the nearest future. The main scientific goal of the NICA/MPD is to explore the mixed phase of quarks, gluons and hadrons in the nucleus-nucleus interactions at the CMS energy range from 4 to $11 \mathrm{GeV}$. To reach the main goal of MPD (Multi-Purpose Detector), the following studies are foreseen for different ions from $\mathrm{H}$ to $\mathrm{Au}$ : event-by-event fluctuation in hadron productions (multiplicity and $\left\langle P_{T}>\right.$ fluctuations), femtoscopic correlation, directed and elliptic flows for various hadrons, multi-strange hyperon production, yield and spectra of hadrons, photon and electron, charge asymmetry and so on.

Nucleus-nucleus collisions with various centralities, with various impact parameters, will be studied at NICA/MPD experiment. For determination of the centrality, many experiments (ATLAS, CMS, FOBOS and others) use Glauber Monte Carlo model. It allows one to determine main parameters of interactions such as the impact parameter $(b)$, a number of interacting nucleons $\left(N_{\text {part }}\right)$, a number of binary collisions $\left(N_{\text {coll }}\right)$, an eccentricity - anisotropy of interaction region, masses of residual nuclei and so on. A well-known Glauber Monte Carlo code [1] created by B. Alver, M. Baker et al. for FOBOS collaboration is used for these purposes. The code is used by many modern experiments at high energies: CMS, ALICE, STAR and others. A simplified Glauber approximation is implemented in the code. We will consider a more correct Glauber approximation which can be applied for beam energy scan program realized at NICA/MPD.

A centrality determination of collisions under the study - determination of impact parameter range, is a key problem in experimental investigations. Usually, a mapping of Glauber calculations and experimental observables $\left(n_{o b s}\right)$ is used for the problem solution. In a simple form, it looks like that: $<n_{\text {obs }}>=\left[\alpha<N_{\text {part }}>+(1-\alpha)<N_{\text {coll }}>\right] * \beta$, where $\alpha$ and $\beta$ are tuned parameters. A question is what

^e-mail: galoyan@1xpub01.jinr.ru 
observable is the most appropriate for this? It maybe a multiplicity of charged particles registered in a detector, or multiplicity of spectator neutrons, or energy carried by spectator nuclear fragments, and so on. We shortly consider them below.

\section{Main ingredients of Glauber approach}

Cross section of new particle production in nucleus-nucleus interactions is given in the Glauber approximation by the well-known expression:

$$
\begin{gathered}
\sigma_{A B}^{p r o d}=\sigma_{A B}^{t o t}-\sigma_{A B}^{e l}-\sigma_{A B}^{q . e l}=\int d^{2} b\left\{1-\prod_{i=1}^{A} \prod_{j=1}^{B}\left(1-p_{i j}\left(\mathbf{b}-\mathbf{s}_{i}+\tau_{j}\right)\right)\right\} . \\
\left|\psi_{A}\left(\mathbf{r}_{1}, \ldots, \mathbf{r}_{A}\right)\right|^{2}\left|\psi_{B}\left(\mathbf{t}_{1}, \ldots, \mathbf{t}_{B}\right)\right|^{2} \prod_{i=1}^{A} d^{3} r_{i} \prod_{i=1}^{B} d^{3} t_{i},
\end{gathered}
$$

where $A$ and $B$ are mass numbers of the nuclei; $\mathbf{b}$ is the impact parameter;

$$
p_{i j}(\mathbf{b})=C_{i} C_{j} \gamma(\mathbf{b})+C_{i} C_{j} \gamma^{*}(\mathbf{b})-\left(C_{i} C_{j}\right)^{2} \gamma(\mathbf{b}) \gamma^{*}(\mathbf{b}) ;
$$

$\gamma(\mathbf{b})$ is an amplitude of elastic nucleon-nucleon scattering in the impact parameter representation; $C_{i}$ is shower enhancement coefficient in the vertex of pomeron-nucleon interaction; $\left\{\mathbf{s}_{i}\right\}, i=1,2, \ldots, A$ and $\left\{\tau_{j}\right\}, j=1,2, \ldots, B$ are sets of coordinates of nucleons of $A$ and $B$ nuclei in the impact parameter plane; $\mathbf{r}_{i}=\left(\mathbf{s}_{i}, z_{i}\right) ; \mathbf{t}_{i}=\left(\tau_{i}, \zeta_{i}\right) ; \psi_{A}$ and $\psi_{B}$ are wave functions of the nuclei in the ground states. At $C_{i}=1$ one has the standard Glauber expression. At $C_{i} \neq 1$ the Gribov's inelastic screenings are taken into account. In this case each nucleon can be in an "active" state with a probability $1 / C$ and $C_{i}=C$, or in a "passive" state with a probability $1-1 / C$.

Equation (1) can be re-written in a form where each term of the expansion can be interpreted as a cross section of processes with fixed multiplicity of nucleon-nucleon collisions:

$$
\begin{aligned}
& \sigma_{A B}^{p r o d}=\int d^{2} b\left\{\sum_{i=1}^{A} \sum_{j=1}^{B} \frac{p_{i j}\left(\mathbf{b}-\mathbf{s}_{i}+\tau_{j}\right)}{1-p_{i j}\left(\mathbf{b}-\mathbf{s}_{i}+\tau_{j}\right)} \prod_{k=1}^{A} \prod_{l=1}^{B}\left(1-p_{k l}\left(\mathbf{b}-\mathbf{s}_{k}+\tau_{l}\right)\right)+\right. \\
&+ \frac{1}{2} \cdot \sum_{\substack{i=1, j=1 \\
l \neq j}}^{A} \sum_{k=1}^{B} \frac{p_{i k}\left(\mathbf{b}-\mathbf{s}_{i}+\tau_{k}\right)}{1-p_{i k}\left(\mathbf{b}-\mathbf{s}_{i}+\tau_{k}\right)} \frac{p_{j k}\left(\mathbf{b}-\mathbf{s}_{j}+\tau_{k}\right)}{1-p_{j k}\left(\mathbf{b}-\mathbf{s}_{j}+\tau_{k}\right)} \cdot \\
&\left.\prod_{l=1}^{A} \prod_{m=1}^{B}\left(1-p_{l m}\left(\mathbf{b}-\mathbf{s}_{l}+\tau_{m}\right)\right)+\ldots\right\} \\
&\left|\psi_{A}\left(\mathbf{r}_{1}, \ldots, \mathbf{r}_{A}\right)\right|^{2}\left|\psi_{B}\left(\mathbf{t}_{1}, \ldots, \mathbf{t}_{B}\right)\right|^{2} \prod_{i=1}^{A} d^{3} r_{i} \prod_{i=1}^{B} d^{3} t_{i} .
\end{aligned}
$$

For example, the first term is a cross section of processes with one inelastic nucleon-nucleon collision. The second term is a cross sections of processes with two inelastic nucleon-nucleon collision, and so on.

A complexity of (1) and (2) prevent their analytical evaluation at a realistic choice of the scattering amplitude and nuclear densities with an exception $A, B \leq 4$. Thus, it was proposed in the papers [2] to use Monte Carlo averaging methods for the aim. 
In particular, in the paper [1] a program code was proposed which uses modern computational tools - the ROOT system. The code is intensively used in experiments at high energies, and is called "Glauber Monte Carlo". Though, the code is not free from some drawbacks: the profile of inelastic nucleon-nucleon collisions is chosen in a simplified form $\left(p_{i j}(\mathbf{b})=\theta\left(\mathbf{r}_{\mathrm{NN}}-|\mathbf{b}|\right), r_{N N}=\sqrt{\sigma_{N N}^{i n} / \pi}\right)$, a set of allowed nuclei is strongly restricted. These mean that one needs to point out working with the code a cross section of inelastic nucleon-nucleon collisions at a given energy of the collisions, and nuclei from a defined list. Some drawbacks of the code were erased in the paper [3].

Recently, we have proposed $[4,5]$ the following parameterization of the nucleon-nucleon elastic scattering amplitude which can be used in the Glauber calculations:

$$
\begin{gathered}
\gamma(b)=A_{1}\left\{\frac{1}{1+e^{(b-\tilde{R}) / d}}+\frac{1}{1+e^{-(b+\tilde{R}) / d}}-1\right\}- \\
-i \rho A_{1} \frac{\tilde{R}^{2} / 2+\pi^{2} d^{2} / 6}{\tilde{R} d}\left\{\frac{e^{-(b-\tilde{R}) / d}}{\left[1+e^{-(b-\tilde{R}) / d}\right]^{2}}+\frac{e^{-(b+\tilde{R}) / d}}{\left[1+e^{-(b+\tilde{R}) / d}\right]^{2}}\right\}- \\
-i \frac{A_{2}}{2 \pi B_{2} 25.64} e^{-b^{2} /\left(2 B_{2} 25.64\right)} \\
\tilde{R}=R+\left(0.07+d+0.2 d^{2}\right) e^{-1.2 R / d} .
\end{gathered}
$$

The real part of the amplitude (3) is the symmetrized Fermi distribution. The imaginary part of the amplitude which is small at high energies has rather complicated form due to usage of dispersion relations in differential forms. The last term of the amplitude is needed for a description of nucleonnucleon elastic scattering at large momentum transfers $\left(|t|>1.75(\mathrm{GeV} / \mathrm{c})^{2}\right)$. Energy dependencies of $\tilde{R}, R, d, A_{1}, A_{2}, B_{2}, \rho$ are given in [6]. The parameterization allows one to describe rather well experimental data on differential cross sections of proton-proton elastic scattering at $\sqrt{s} \geq 3 \mathrm{GeV}$ [5].

Important structure elements of (1) and (2) are squared modules of the nuclear wave functions in the ground states. Very often they are represented as:

$$
\left|\psi_{A}\left(\mathbf{r}_{1}, \ldots, \mathbf{r}_{A}\right)\right|^{2}=\left[\prod_{i=1}^{A} \rho_{A}\left(\mathbf{r}_{\mathbf{i}}\right)\right] \delta\left(\frac{1}{A} \sum_{i=1}^{A} \mathbf{r}_{\mathbf{i}}\right)
$$

A systematical analysis of electron-nuclear data and a comparison with theoretical calculations (DHB approximation) have been done in the paper [7] for an extraction of one-particle densities of nuclei $\left(\rho_{A}\right)$. As a result, the densities were parameterized in the standard form:

$$
\rho_{A}(r)=\frac{\rho_{0}}{1+e^{\frac{r-R_{A}}{d_{A}}}}, \quad R_{A}=1.31 A^{1 / 3}-0.84 \quad[\mathrm{fm}], \quad d_{A}=0.5 \quad[\mathrm{fm}] .
$$

The paper [7] is well-known in low and intermediate energy physics, especially in physics of exotic nuclei because parameterizations of one-particle densities of protons and neutrons in nuclei were also presented in the paper.

All specifications mentioned above were introduced in our Monte Carlo program of Glauber calculations. Results of calculations with the code are presented below.

Figure 1a shows dependencies of average multiplicities of the participating nucleons on a centrality of $\mathrm{Au}+\mathrm{Au}$ collisions at $\sqrt{s_{N N}}=9$ and $200 \mathrm{GeV}$ (closed and open points, respectively). Dispersions of the quantities are presented as "experimental error bars". As it is seen, the multiplicities of the participating nucleons at low and high energies differ in $3-5 \%$. It is explained by the fact that high 
energy collisions differ from low energy one only due to larger radius of $N N$ interactions from geometrical point of view. The radii are small compared with nuclear sizes. Though, the change of the radius at a transition from low to high energies has significant influence on the average multiplicities of the binary collisions (see figure 1b) and the anisotropy of nuclear interaction region (see figure 1c).
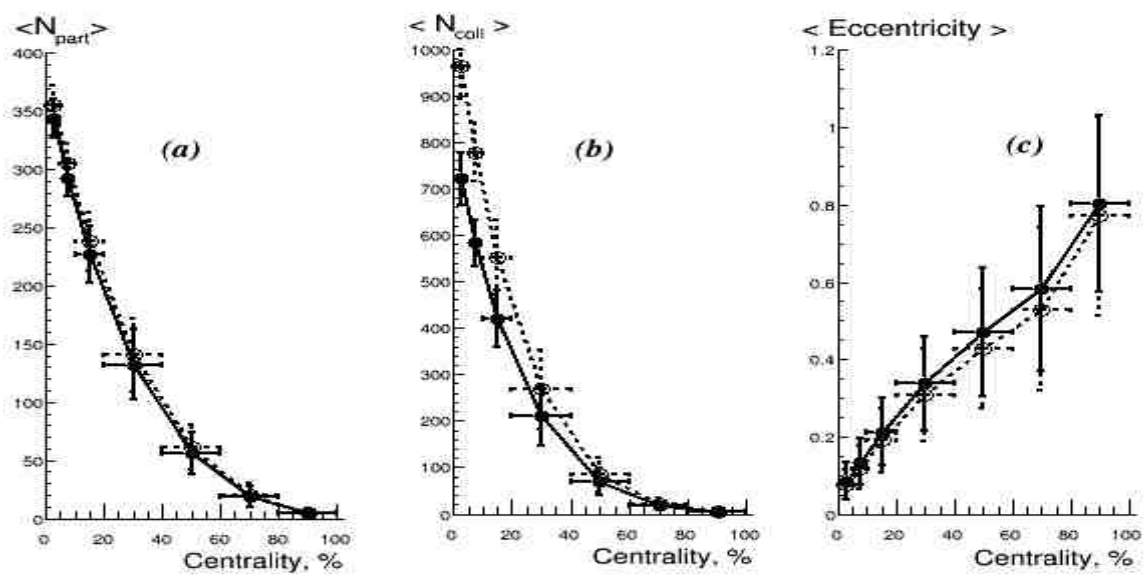

Figure 1. Mean multiplicities of the participating nucleons, binary collisions and the eccentricity as functions of the centrality (figures a, b and c, respectively). Dispersions of the quantities are presented as error bars.

Usually, events of nucleus-nucleus interactions are subdivided on the following centrality classes: $0-5,5-10,10-20,20-40,40-60,60-80,80-100 \%$ at experimental studies of super high energies. As it is seen in Figs. 1a and 1b, distributions on multiplicities of the participating nucleons and the binary collisions are inessential overlapping at high and low energies in neighboring intervals of the centrality at such subdivisions. Thus, the corresponding quantities for neighboring intervals $-\left\langle N_{\text {part }}\right\rangle$ and $<N_{\text {bin }}>$ can be used for revelations of scaling properties of the interactions and the jet quenching in the collisions. Such applications become problematic at a more narrow interval subdivision due to a possible strong overlap of the distributions. As it is also seen, distributions on anisotropy of the interaction regions are strongly overlapping at all centrality classes (see figure 1c).

The anisotropy of the interaction region is characterized by the eccentricity in the last decade $\varepsilon_{\text {part. }}=\left(<y^{2}>-<x^{2}>\right) /\left(<y^{2}>+<x^{2}>\right)$, where $x$ and $y$ are coordinates of a participating nucleon in the impact parameter plane counted from a center of the interaction region. We have used the definition in our calculations. $\varepsilon_{\text {part }}$ in super high energy interactions is proportional to a magnitude of an elliptic flow which is unambiguously connected with the properties of the quark-gluon plasma. One can expect a violation of the regularity and strong fluctuations of the collective flows at low energies and at an appearance of the mixed phase. Studies of such fluctuations are topics of modern experimental efforts of the last time.

\section{Choose of observables for centrality determination}

The geometrical property of interactions cannot be measured directly. They must be connected with experimental observables such as a multiplicity of charged particles in a detector, or an energy carried by spectator nuclear fragments measured by Zero Degree Calorimeter. Possible locations of the 
detectors are shown in figure 2 by rectangles. We present in figure 2 rapidity distributions of particles produced in $\mathrm{Au}+\mathrm{Au}$ interactions at $\sqrt{s_{N N}}=10 \mathrm{GeV}$ predicted by UrQMD [8], QGSM [9], and FTF [10] models.
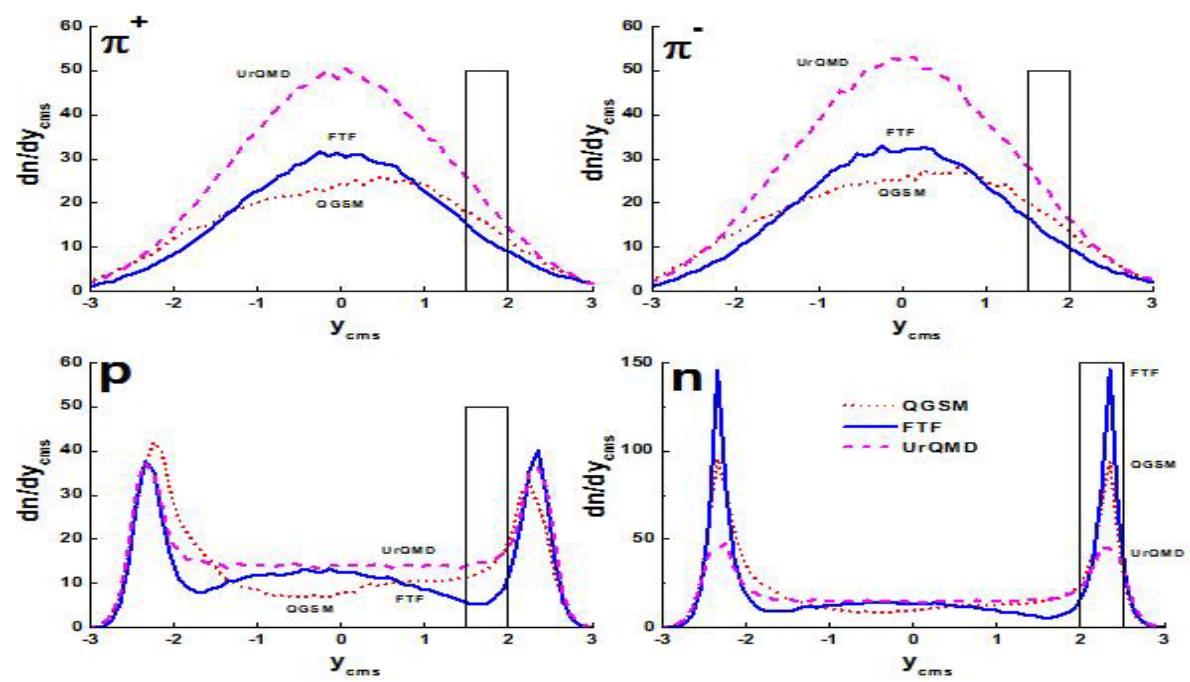

Figure 2. Rapidity distributions of hadrons in $\mathrm{Au}+\mathrm{Au}$ interactions at $\sqrt{s_{N N}}=10 \mathrm{GeV}$. Solid, dashed and dotted lines are FTF, UrQMD and QGSM model calculations, respectively.

As it is seen, the model predictions are in a disagreement with each other. UrQMD predicts the largest production of charged pi-mesons in central region. QGSM predicts the low multiplicity of produced mesons. FTF gives intermediate results. The FTF, UrQMD and QGSM predictions for proton production in $\mathrm{Au}+\mathrm{Au}$ interactions at $10 \mathrm{GeV} /$ per $\mathrm{NN}$ collision are close to each other.

However, UrQMD gives small number of evaporated neutrons as seen in the bottom right figure. To obtain the calculation results, we enlarged the UrQMD by the excitation-de-excitation model. We use for simulation of the de-excitation the well-known Statistical Multi-fragmentation Model (SMM).

Production of nuclear fragment is implemented in the QGSM model.

FTF gives the largest number of produced neutrons in fragmentation region. We checked the FTF predictions for neutron production in nucleus-nucleus interactions in [11].

\section{Conclusion}

- According to our calculations, the geometrical properties of nucleus-nucleus interactions at RHIC and NICA energies are quite close to each other.

- Predictions of various Monte Carlo models for meson production in AA interactions are varied in the range $\pm 50 \%$ for $E_{c m s}=5-10 \mathrm{GeV}$. A large disagreement between the model predictions is observed for spectator neutron productions.

- According to the models, largest particle density must be for neutrons in very narrow interval of rapidity. This predictions can be used for design of centrality detector. 


\section{References}

[1] B. Alver, M. Baker, C. Loizides and P. Steinberg, arXiv:0805.4411 [nucl-exp] (2005)

[2] A.M. Zadorozhnyi, V.V. Uzhinsky and S.Yu. Shmakov, Comp. Phys. Commun. 54, 125 (1989)

[3] W. Broniowski, M. Rybczynski and P. Bozek, Comp. Phys. Commun. 180, 69 (2009)

[4] V. Uzhinsky and A. Galoyan, JETP Lett. 94, 499 (2011). Pisma v Zh.ETP 94, 539 (2011)

[5] V. Uzhinsky and A. Galoyan, arXiv:1111.4984 [hep-ph] (2011). arXiv:1210.7338 [hep-ph] (2012)

[6] A.S. Galoyan and V.V. Uzhinsky, Phys. Part. Nucl. Lett. 12, 166 (2015)

[7] L.C. Chamon et al., Phys. Rev. C 66, 014610 (2002)

[8] S.A. Bass et al., Prog. Part. Nucl. Phys. 41, 225 (1998). M. Bleicher et al., J. Phys. G 25, 1859 (1999)

[9] V.D. Toneev, N.S. Amelin, K.K. Gudima, and S.Yu. Sivoklokov, Nucl. Phys. A 519, 463 (1990)

[10] http://geant4.cern.ch/support/userdocuments.shtml/PhysicsReferenceManual.pdf

[11] A. Galoyan, A. Ribon and V. Uzhinsky, JETP Letters 102, 324 (2015) 